\title{
A Training Effect by Three-Month Watering on the Frontal Pole Activation of Healthy Older Adults
}

\author{
Masahiro Toyoda $^{1 *}$, Yuko Yokota1, Hiroyuki Kikukawa ${ }^{2}$ \\ ${ }^{1}$ Graduate School of Landscape Design and Management, University of Hyogo, Awaji, Japan \\ ${ }^{2}$ Graduate School of Agriculture, Kyoto University, Kyoto, Japan \\ Email: ${ }^{\star}$ masahiro_toyoda@awaji.ac.jp
}

How to cite this paper: Toyoda, M., Yokota1, Y. and Kikukawa, H. (2020) A Training Effect by Three-Month Watering on the Frontal Pole Activation of Healthy Older Adults. Journal of Behavioral and Brain Science, 10, 265-285.

https://doi.org/10.4236/jbbs.2020.106017

Received: March 12, 2020

Accepted: June 16, 2020

Published: June 19, 2020

Copyright $\odot 2020$ by author(s) and Scientific Research Publishing Inc. This work is licensed under the Creative Commons Attribution International License (CC BY 4.0).

http://creativecommons.org/licenses/by/4.0/

\begin{abstract}
The effectiveness of training prefrontal lobe function for suppressing cognitive decline or improving cognitive function has been reported. Some epidemiological studies suggested that gardening activities can contribute to suppression of cognitive decline. In our previous studies using functional Near-Infrared Spectroscopy (fNIRS), we reported the possibility of frontal pole (FP) activation of healthy older adults during gardening activities in the quasi-laboratory setting. The objective of this study was to examine the impacts of a three-month continual practice of the watering task in the actual gardening settings on the FP activation of healthy older adults. The participants were 18 right-handed healthy older adults without a diagnosis of mild cognitive impairment or dementia ( $M=71.7$ years \pm SD 5.0, range: 63 - 84 years) who lived independently at home. We asked the participants to continue watering at home for three months in the same way as the research task in the fNIRS measurement. After a three-month intervention, the participants' task performance improved compared with that of pre-intervention. In addition, the Oxy-Hb values in the right lateral FP during the third trial in the post-intervention measurement were significantly greater compared with the pre-intervention measurement. This study suggested that even a gardening activity with a small number of processes such as watering, if the activity is continued for a certain period of time, can suppress the decline of the FP activation of healthy older adults. Combination of various gardening activities has the potential to be a further cognitive training for activating the FP.
\end{abstract}

\section{Keywords}

Cognitive Training, Dementia Prevention, Functional Near-Infrared Spectroscopy (fNIRS), Stimulus-Independent (SI) Thought, 
Stimulus-Oriented (SO) Attention

\section{Introduction}

According to the fact sheet on dementia published by World Health Organization on Sept. 19, 2019, approximately 50 million people live with dementia as of 2015 throughout the world, and there are nearly 10 million new cases every year. In addition, the number of people with dementia is estimated to reach 82 million in 2030 and to triple in 2050 [1]. Dementia prevention is an urgent global issue. Decline of cognitive and executive function occurs not only in people with Alzheimer's disease or Mild Cognitive Impairment (MCI), but in healthy older adults with aging. Some previous studies have reported a decline of the working memory [2], a decrease of the blood flow in the dorsolateral prefrontal cortex (DLPFC) [3] and so on as the reason for cognitive decline with aging. Meanwhile, other studies have reported the effectiveness of the activities stimulating and training prefrontal lobe function for suppressing cognitive decline or improving cognitive function in various populations including people with MCI [4].

Some epidemiological studies reported that physical, social and leisure activities including gardening are effective [5] [6] [7] [8]. Furthermore, Jedrziewski, Ewbank, Wang, and Trojanowski reported additional benefits gained from socialization and cognitive stimulation through different types of exercises including gardening/yard work [7].

We have conducted a series of studies to validate a hypothesis that the kinetic features of gardening activities stimulate the brain and contribute to reduction of the risk of dementia. One of the most noteworthy characteristics of gardening is that the gardening includes motor programming for repetition of the same motion.

In our first functional Near-Infrared Spectroscopy (fNIRS) study targeting older adults over 60, we compared the activation of the prefrontal area in the brain using three tasks of the Frontal Assessment Battery (FAB). The three tasks were a motor programming task (FAB 3), a sensitivity-to-interference task (FAB 4 ) and an inhibitory control task (FAB 5). The study indicated that the activation of the frontal pole (FP) and the dorsolateral prefrontal cortex (DLPFC) by a motor programming task (FAB 3) was greater than or comparable with that of other two tasks [9].

In our second fNIRS study, we examined the activation of the FP and the DLPFC of the participants [healthy younger adults (age range 20 - 59 years) and healthy older adults (age range 60 - 88 years)] using five typical gardening tasks as research tasks and the FAB 3 task as a control task. The five gardening tasks were seeding, thinning, planting, weeding and watering. Each task was repeated three times. The group of younger adults showed the activation compatible with or greater than that of the FAB 3 task in the five gardening tasks [10]. This suggested that gardening activities could induce greater activation of the prefrontal 
area than a simple motor programming task like the FAB 3 task. On the other hand, the group of older adults showed greater activation in the DLPFC during the seeding task and in the FP during the watering task compared with that during the FAB 3 task.

In our third fNIRS study, we focused on two gardening tasks that had induced greater activation than the FAB 3 task in the prefrontal cortex (PFC) of older adults in our second fNIRS study. Increasing the number of trials from three to five times, we examined the activation in the FP of healthy older adults during the seeding task, the watering task and the FAB 3 task. The results showed a decreased activation in the FP while the FAB 3 task was repeated five times, whereas the activation during the gardening tasks seemed to be hard to decrease even though the trials increased [11]. The presumable cause for the FP having been activated even in the fifth trial was that the participants had needed to pay attention to two external stimuli (i.e. visual information and somatosensory information) at the same time in order to perform the gardening tasks accurately. Furthermore, attention to external stimuli and internal thought were repeated while the participants were performing the gardening tasks. These are related to that the role of the medial and the lateral FP were necessary during the gardening tasks. The study showed that a certain of motion-related elements included in gardening activities could activate the FP repeatedly in the quasi-laboratory setting.

However, a problem remained: Will the activation in the FP be maintained even if the gardening activity is repeated over a prolonged period in the actual setting? Therefore, the objective of this study was to examine the FP activation of the healthy older adults after a three-month continual performance of the same gardening task as the research task in the actual gardening setting.

\section{Materials and Methods}

All participants received a detailed explanation of the study such as objectives, methods, prospective risk, and privacy policy prior to the study and provided written informed consent. The research protocol was approved by the Ethics Committee in the Graduate School of Landscape Design and Management, the University of Hyogo in November 2015.

\subsection{Participants}

This study was conducted from April 15 to July 12, 2016. We recruited a volunteer group of the healthy, right-handed older adults to participate in the study through the Social Welfare Council in Tatsuno City, Hyogo Prefecture, Japan. Eighteen members of the Asatomi senior citizen's club in Tatsuno City offered their cooperation for the study. All of them lived independently at home and had not been received a diagnosis of having mild cognitive impairment or dementia. The participants were 18 right-handed healthy older adults $(M=71.7$ years $\pm S D$ 5.0, range: $63-84$ years $)$ of which two were males $(M=74.5$ years \pm SD 0.71 , 
range: 74 - 75 years) and 16 were females $(M=71.4$ years \pm SD 5.3, range: 63 84 years). Their right-handedness was confirmed in the oral interview and through observation of their hand motion during the fNIRS measurements.

\subsection{Protocol}

Watering seemed to be appropriate as a gardening task for the participants easy to do and continue at home. We requested the participants to continue the watering activity at home for three months and measured the blood flow in the FP during the watering task using fNIRS before and after the intervention period. The way of watering at home was the same as the watering task at measurement (Figure 1).

\subsection{Functional Near-Infrared Spectroscopy (fNIRS) Measurements}

\subsubsection{Device}

fNIRS is a noninvasive tool to examine brain activity while the participants are performing activities in a sitting posture. We measured relative changes in the concentration of oxygenated hemoglobin $(\mathrm{Oxy}-\mathrm{Hb})$, deoxygenated hemoglobin (Deoxy-Hb), and total hemoglobin (Total-Hb) during the tasks by using an fNIRS equipment, Spectratech OEG-16, (Spectratech Inc., Tokyo). The equipment has 16 simultaneous measurement channels and uses two wavelengths (770 $\mathrm{nm}$ and $840 \mathrm{~nm}$ ) of near-infrared light. The constituents of an OEG-16 sensor band are light emitting parts of six built-in type LEDs with two waves and light receiving parts of six photo diodes. Each channel $(\mathrm{CH})$ (i.e. measuring point) consists of a pair of optodes-one emitter and one detector-with a distance between them of $30 \mathrm{~mm}$ (Figure 2). An fNIRS optode is an optical sensor device to measure the local changes in Oxy-Hb, Deoxy-Hb and Total-Hb in the PFC. Absorption of near-infrared light was measured with a sampling interval of 0.65 seconds. In attaching the head unit onto the participant's forehead, the center of

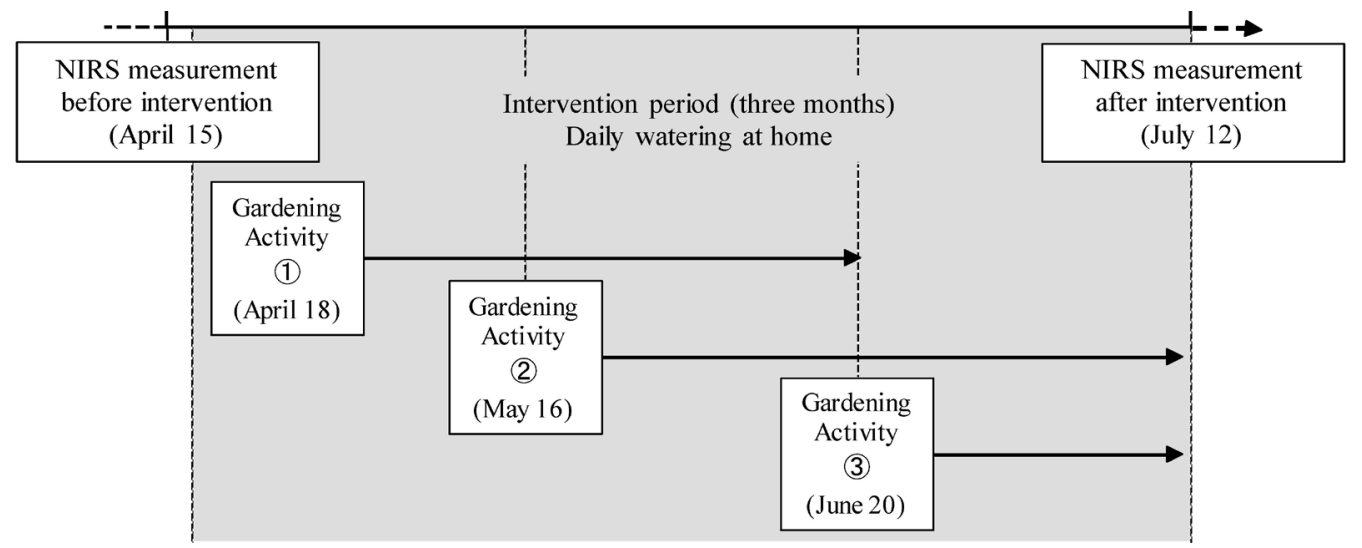

Figure 1. Protocol of the study. We conducted the fNIRS measurement before and after the intervention period. We provided three sessions of gardening activities during the intervention period. After each session, the participants brought home the cell tray where they had sowed or had planted cuttings in order to carry on watering at home. fNIRS = functional Near Infrared Spectroscopy. 


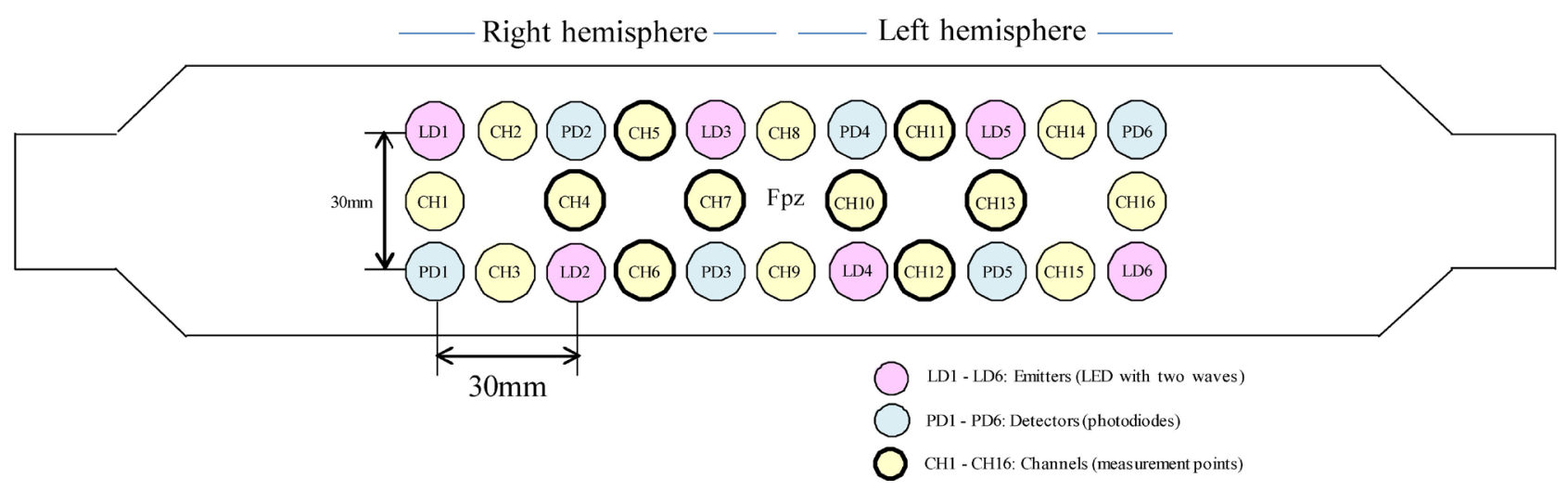

Figure 2. Location of 12 optodes and 16 measurement points (channels) in the OEG-16 head module. One channel consists of 1 emitter and 1 detector at a distance of $30 \mathrm{~mm}$ from each other. The head unit was attached to the forehead of the participant according to the International 10 - 20 system generally used for electroencephalography (EEG). In this study, we focused on the channels reflecting the activity of the frontal pole (FP) shown in heavy line circles. $\mathrm{CH} 7$ and $\mathrm{CH} 10$ reflect the activity in the medial FP and CHs $4-6$ and $\mathrm{CHs} 11-13$ reflect the activity in the lateral FP.

the sensor band was placed on the Fpz according to the International $10-20$ system, a standard for electroencephalography (EEG) electrode positioning [12]. The sensor band was covered with a headband in order to make sure that all optodes contacted the skin of the forehead of the participant. We used the variations in the Oxy- $\mathrm{Hb}$ concentration as indicators of changes in the regional cerebral blood flow, because Oxy-Hb was considered to be more sensitive than Deoxy-Hb as a parameter for measuring the blood flow relevant to PFC activation, as Hoshi, Kobayashi, and Tamura noted [13].

The regions measured by fNIRS in our study included the FP, the DLPFC, the inferior frontal gyrus (including the orbitofrontal cortex), the pars triangularis Broca's area, and the frontal eye field. In the present study, we focused on the FP and used the data of $\mathrm{CH} 4, \mathrm{CH} 5$ and $\mathrm{CH} 6$ as the right lateral $\mathrm{FP}, \mathrm{CH} 7$ as the right medial FP, $\mathrm{CH} 10$ as the left medial FP, and $\mathrm{CH} 11, \mathrm{CH} 12$ and $\mathrm{CH} 13$ as the left lateral FP.

\subsubsection{Measurements}

As shown in Figure 1, the fNIRS measurements were conducted just before and after the intervention period in a quiet room in the building of Tatsuno Social Welfare Center, Tatsuno City, Hyogo Prefecture, Japan. The room size was approximately 60 square meters. The measurement environment was as follows: the first measurement on April 15 (room temperature: $19.1^{\circ} \mathrm{C}-21.8^{\circ} \mathrm{C}$, humidity: $50.0 \%-56.0 \%$ ) and the second measurement on July 12 (room temperature: $23.0^{\circ} \mathrm{C}-25.8^{\circ} \mathrm{C}$, humidity: $58.0 \%-63.0 \%$ ) in 2016 . Each participant entered the room individually and performed tasks in a sitting posture with fNIRS optodes positioned on the forehead.

The protocol of the fNIRS measurement was as follows: instructions on the watering task ( 45 seconds), the baseline period ( 15 seconds) and [the watering task ( 15 seconds) and rest ( 15 seconds) $] \times 3$ sets. The instructions on the watering task in the pre-intervention measurement included demonstration by the 
examiner and practice by the participant. The number of the practice by the participant was once. The practice in the instructions was skipped in the post-intervention measurement. During task performance, the participants were instructed not to move their heads widely. During the baseline period and the rest period, participants were requested to gaze blankly at a double circle on a piece of white paper in front of them located at a distance of one meter so they could recover enough to be stable (Figure 3 ).

\subsection{Tasks}

\subsubsection{Watering Task}

The watering task was conducted using a black cell tray with 25 cells (five-by-five-cell) filled with culture soil by a research assistant in advance. The level of the soil in each cell was $10 \mathrm{~mm}$ lower than the brim to provide enough watering space.

We provided a cylindrical plastic bottle of $500 \mathrm{ml}$ in capacity $(65 \mathrm{~mm}$ across $\times$ $220 \mathrm{~mm}$ high) with a plastic sprinkling nozzle attached. We explained to the participants that they did not need to squeeze the bottle because water ran out through the nozzle just by tilting the bottle. The participant was instructed to start watering from the top left cell, move rightward to the end of the first row, proceed to the left-most cell of the next lower row and move rightward to the end of the row. The participant repeated this process to the bottom right cell (Figure 3).

\subsubsection{Provision of Daily Activity at Home}

To investigate impacts of the routine watering on the FP activation after a certain period of time, we asked the participants to continue watering at home in the same way as the watering task in the fNIRS measurement for three months. Taking the burden on the participants into account, we decided the watering duration of three months. However, some motivation seemed to be necessary for the participants to continue the watering activity at home for three months. Therefore, in the course of the study period, we provided the participants with

\begin{tabular}{|c|c|c|c|c|c|c|c|}
\hline \multirow[b]{2}{*}{$\begin{array}{l}\text { Instructions } \\
\text { and practice }\end{array}$} & \multicolumn{7}{|c|}{ Measurement } \\
\hline & $\begin{array}{c}\text { Baseline } \\
\text { period }\end{array}$ & $\begin{array}{c}\text { 1st trial } \\
\text { Task }\end{array}$ & Rest & $\begin{array}{c}\text { 2nd trial } \\
\text { Task }\end{array}$ & Rest & $\begin{array}{c}\text { 3rd trial } \\
\text { Task }\end{array}$ & Rest \\
\hline $45(\mathrm{sec})$ & 15 & 15 & 15 & 15 & 15 & 15 & 15 \\
\hline
\end{tabular}
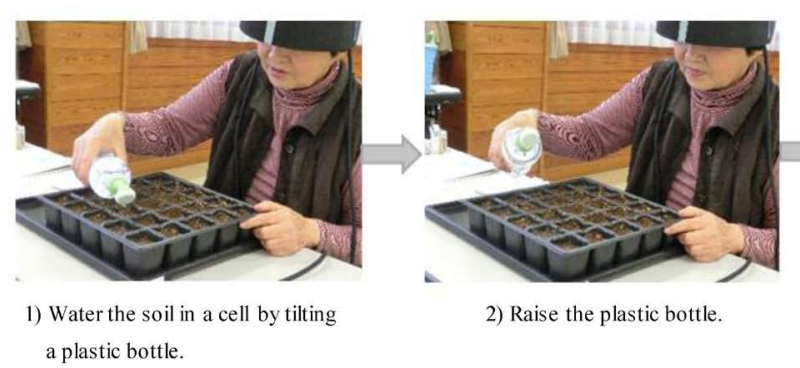

2) Raise the plastic bottle.

a plastic bottle.

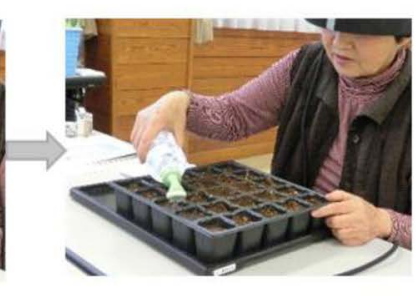

3) Move to the next cell and water the soil.

Figure 3. The protocol of the fNIRS measurement and the process of a watering task. A 15-second trial of the watering task was conducted three times in the fNIRS measurement. 
three gardening programs requiring continuous watering at home after the activity. We held three group gardening sessions for the participants on April 18, May 16 and June 20. These gardening sessions created the participants' need to take care of the plants at home.

The activities provided in the gardening sessions were as follows: April-Seeding of salad rocket (Eruca), May-Seeding of radioxenon (Raphanus sativus var. sativus), June-Stem cutting of herbs [lavender (Lavandula angustifolia), rosemary (Rosmarinus officinalis), and stevia (Stevia rebaudiana)]. Black cell trays with 25 cells (five-by-five-cell) were used in all activities. Each participant brought his/her own cell tray back home each time and was instructed to water the soil when the surface of the soil in the cells got dry in the same way of the watering task in the fNIRS measurement. We lent each participant a plastic bottle attached to a sprinkling nozzle that used in the fNIRS measurement.

\subsection{Data Analysis}

Statistical analyses were performed using Excel 2010 (Microsoft, USA) with the add-in software SSRI, Version 1.02, 2012 (Social Survey Research Information Co., Ltd.).

\subsubsection{Task Performance of the Watering Task}

As evaluation of the watering task performance, the examiner counted the number of times of watering (i.e. the number of watered cells) during the 15 -second task for each trial in the fNIRS measurements. Unfinished cells were not counted.

For comparison among three trials within the task performance in the preand the post-intervention fNIRS measurements (i.e. intragroup comparison), Scheffe's method of paired comparisons was conducted after the Friedman test $(p<0.05)$.

For comparison between the corresponding numbered trials in the pre- and the post-intervention fNIRS measurements (i.e. intergroup comparison), two-sided Wilcoxon Signed-Rank test was conducted $(p<0.05)$.

\subsubsection{Cerebral Blood Flow during the Watering Task}

Sakatani, Xie, Lichty, Li, and Zuo had reported that the fNIRS parameters tended to return toward the preactivation baseline after reaching the maximum value, or to fluctuate during the task, possibly because of attention changes [14].

Burgess, Gilbert, and Dumontheil proposed:

Rostral prefrontal cortex (PFC: approximating area 10) supports a cognitive system that facilitates either stimulus-oriented (SO) or stimulus-independent (SI) attending. SO attending is the behavior required to concentrate on current sensory input, whereas SI attending is the mental processing that accompanies self-generated or self-maintained thought [15].

Gilbert, Frith, and Burgess reported transient activity in the right lateral rostral PFC by a switch between SO and SI thought [16]. Concerning data analysis 
of the fNIRS measurements, we used the maximum Oxy-Hb value during the task (i.e. the highest level from the baseline) to investigate the variation related to the switching of attention. We also used the mean $\mathrm{Oxy}-\mathrm{Hb}$ value during the task to investigate the continuity of the FP activation.

In the data analyses, the blood flow change associated with the brain function activity was extracted by separating fNIRS signals into functional and systemic components using "Hemodynamics modality separation (HMS) method". The method was developed by the National Institute of Advanced Industrial Science and Technology for Spectratech OEG series fNIRS equipments [17] [18].

For comparison of Oxy-Hb values among three trials within the pre- and the post-intervention fNIRS measurements (i.e. intragroup comparison), Scheffe's method of paired comparisons was conducted after the Friedman test $(p<0.05)$.

For comparison of Oxy-Hb values between the corresponding numbered trials in the pre- and the post-intervention fNIRS measurements (i.e. intergroup comparison), two-sided Wilcoxon Signed-Rank test was conducted $(p<0.05)$.

\section{Results}

\subsection{Task Performance of the Watering Task}

\subsubsection{Estimation of the Total Number of Times of Watering at Home in the Intervention Period}

Each participant brought home a cell tray of 25 cells, where he/she sowed or planted cuttings in the group activity in April, May, and June respectively, and carried on watering to cultivate his/her own plants at home. We calculated the total number of times of watering per participant in the intervention period, assuming that a participant performed watering at least once every three days. First, we calculated the number of times of watering in the following three periods, and then, summed them up to calculate the total number of times of watering in the intervention period.

The number of times of watering per participant in each period

$=($ the number of cells $) \times($ the number of cell trays $) \times($ the number of days $) \times 1 / 3$

From April 18 to May 15 (28 days):

$$
25 \times 1 \times 28 \times 1 / 3=233.3 \text { (times). }
$$

From May 16 to June 19 (35 days):

$$
25 \times 2 \times 35 \times 1 / 3=583.3 \text { (times). }
$$

From June 20 to July 12 (23 days):

$$
25 \times 2 \times 23 \times 1 / 3=383.3 \text { (times). }
$$

During the period between May 16 and June 19, the participants performed watering to two cell trays sown seeds in the gardening activities on April 18 and May 16. During the period between June 20 and July 12, the participants also performed watering to two cell trays sown seeds on May 16 and planted cuttings on June 20. The total number of times of watering per participant in the inter- 
vention period was calculated by adding Equation (1), Equation (2) and Equation (3):

$$
233.3+583.3+383.3=1199.9 \text { (times). }
$$

The estimated number of times of watering at home was 1199.9 times in total during the three-month intervention period.

\subsubsection{Intragroup Comparison of Task Performance}

Comparison of the performance of the watering task among three trials in the pre-intervention measurement showed an upward increase in the median of the number of times of watering: 6.0 (the first trial), 6.5 (the second trial), and 7.0 (the third trial). In addition, the results of Scheffe's method of paired comparisons after the Friedman test $(p<0.05)$ demonstrated that the number of times of watering in the second trial and the third trial were significantly larger than that in the first trial $(p=0.0027$ and $p=0.0027$ respectively). There was no significant difference between the second and the third trials. The median of the number of times of watering among three trials in the post-intervention measurement showed an upward increase as is the case with the trials in the pre-intervention measurement: 7.5 (the first trial), 8.5 (the second trial), and 9.0 (the third trial). Scheffe's method of paired comparisons after the Friedman test demonstrated that the number of times of watering in the third trial was significantly larger than that in the first trial $(p=0.0360)$. There was no significant difference between the second and the third trials (Figure 4).

\subsubsection{Intergroup Comparison of Task Performance}

The Wilcoxon Signed-Rank test showed that the number of times of watering in
Pre-intervention
Post-intervention

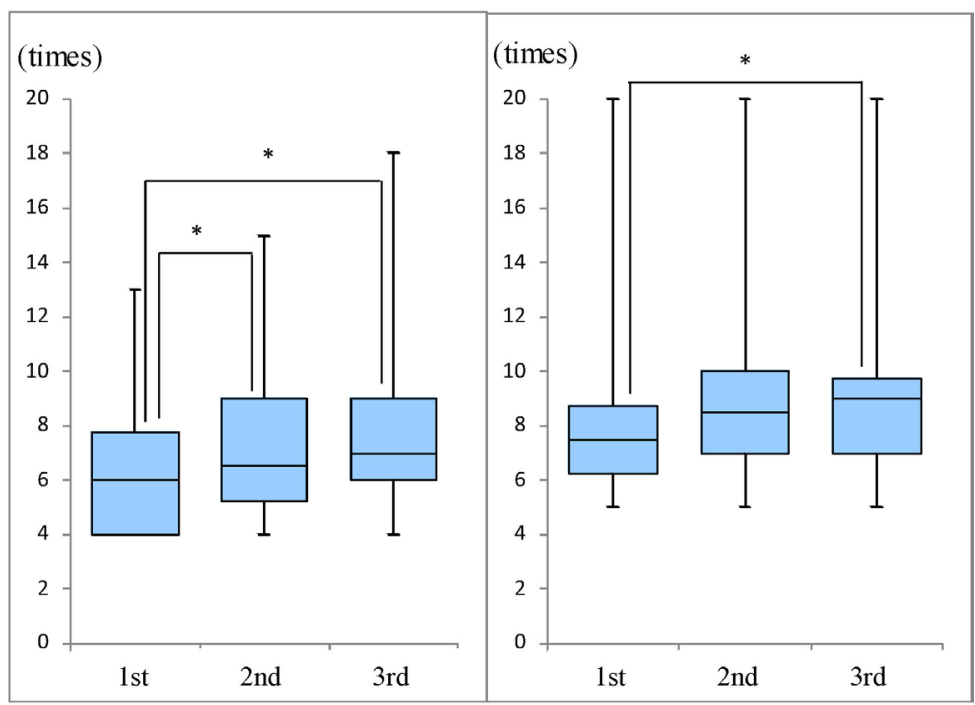

Figure 4. Comparison of the task performance among three trials in the pre-intervention and the post-intervention measurements. Task performance was measured by counting the number of times of watering (i.e. the number of watered cells) per 15-second trial. The longitudinal axis represents the number of times of watering: ${ }^{\star} p<0.05$. 
the first trial in the post-intervention measurement was significantly larger than that in the pre-intervention measurement ( $p=0.0138)$ (Figure 5). Concerning the second trials and the third trials, there were no significant differences detected between the pre- and the post-intervention measurements.

\subsection{Cerebral Blood Flow during the Watering Task}

\subsubsection{Intragroup Comparison of the Maximum Oxy-Hb Values among} Three Trials in the Pre- and Post-Intervention fNIRS Measurements In the pre-intervention fNIRS measurement, the maximum $\mathrm{Oxy}-\mathrm{Hb}$ values tended to decrease in the second and/or the third trial(s). The measurement points and the trial(s) where the maximum $\mathrm{Oxy}-\mathrm{Hb}$ values significantly decreased compared with the first trial were as follows: $\mathrm{CH} 4$ (the second trial: $p=$ 0.0439), $\mathrm{CH} 5$ (the second trial: $p=0.0286$ ), and $\mathrm{CH} 6$ (the second trial: $p=$ 0.0066 and the third trial: $p=0.0181$ ) in the right lateral FP (Figure 6). There was no significant decrease among three trials in the medial FP and the left lateral FP.

In the post-intervention fNIRS measurement, the maximum $\mathrm{Oxy}-\mathrm{Hb}$ values showed the same decreasing tendency as the pre-intervention. The measurement points and the trial(s) where the maximum $\mathrm{Oxy}-\mathrm{Hb}$ values significantly decreased compared with the first trial were as follows: $\mathrm{CH} 4$ (the second trial: $p=$ 0.0181 ) in the right lateral FP; $\mathrm{CH} 7$ (the second trial: $p=0.0286$ ) in the right medial FP and at $\mathrm{CH} 10$ (the second trial: $p=0.0439$ ) in the left medial FP; $\mathrm{CH}$ 11 (the third trial: $p=0.0181$ ), and $\mathrm{CH} 13$ (the second trial: $p=0.0066$ and the third trial: $p=0.0181$ ) in the left lateral FP (Figure 6).

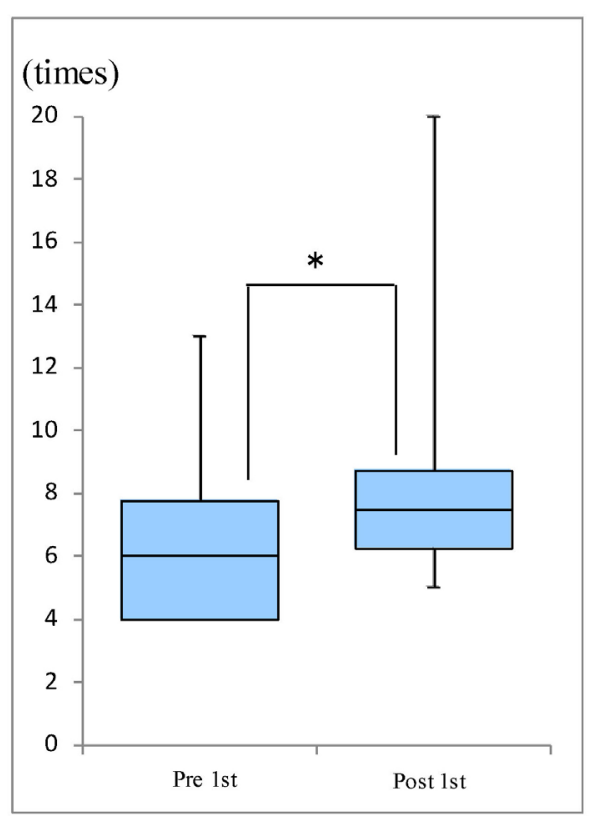

Figure 5. Comparison of the task performance between the first trials in the pre-intervention and the post-intervention measurements. Task performance was measured by counting the number of times of watering (i.e. the number of watered cells) per 15-second trial. The longitudinal axis represents the number of times of watering: ${ }^{\star} p<0.05$. 


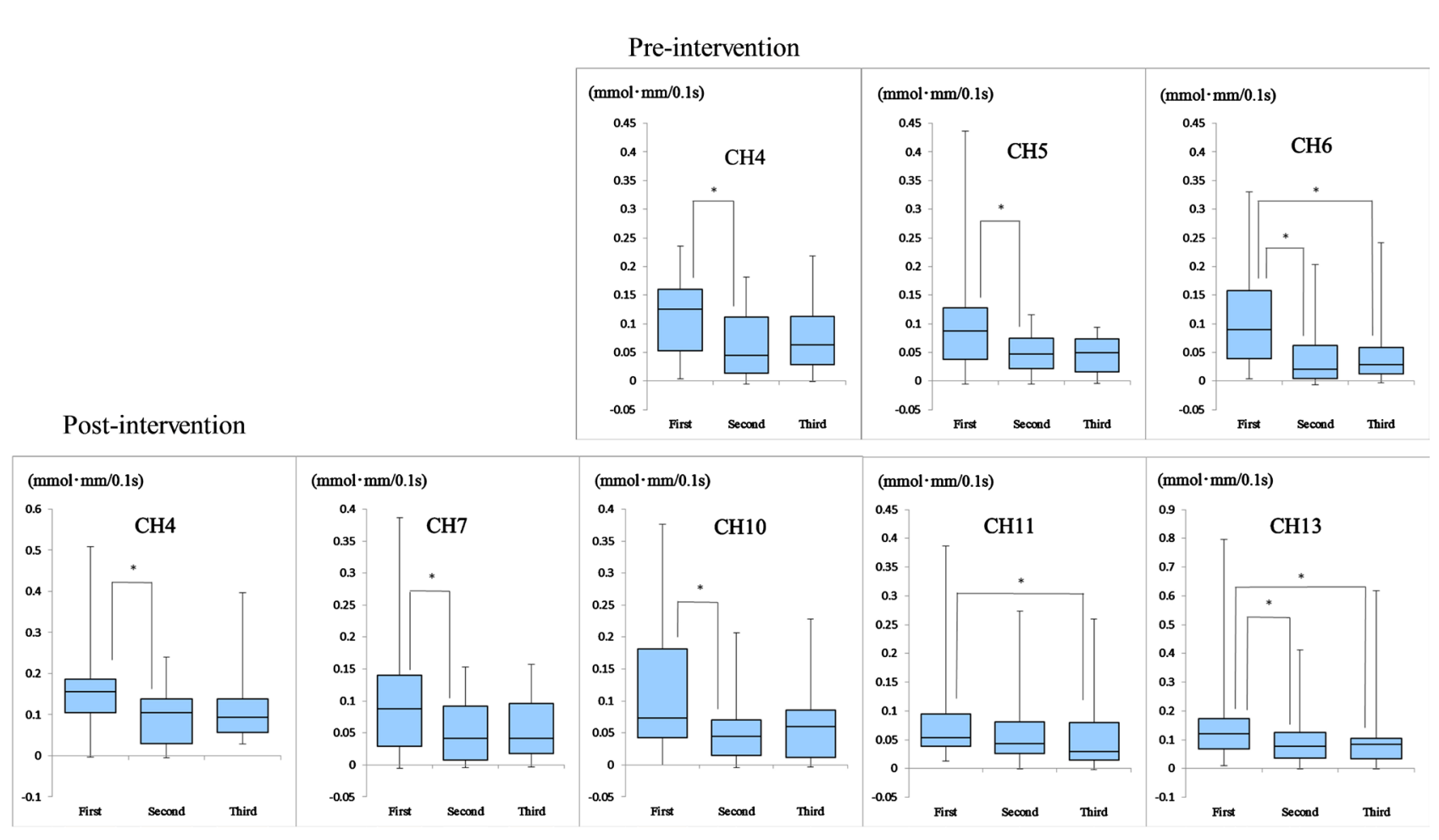

Figure 6. Comparison of the maximum $\mathrm{Oxy}-\mathrm{Hb}$ values during the watering task among three trials in the pre- and the post-intervention measurements. Boxplots on the upper column show the measurement points that demonstrated significant changes in the pre-intervention measurement and boxplots on the lower column show those in the post-intervention measurement: ${ }^{\star} p<0.05 . \mathrm{CH} 4, \mathrm{CH} 5$, and $\mathrm{CH} 6$ reflect the right lateral $\mathrm{FP}, \mathrm{CH} 11$ and $\mathrm{CH} 13$ reflect the left lateral FP. $\mathrm{CH} 7$ reflects the right medial FP and $\mathrm{CH} 10$ reflects the left medial FP. The longitudinal axis represents Oxy-Hb concentration.

\subsubsection{Intragroup Comparison of Mean Oxy-Hb Values among Three Trials in the Pre- and Post-Intervention fNIRS Measurements}

In the pre-intervention fNIRS measurement, the mean Oxy-Hb values tended to decrease in the second and/or the third trial(s). The measurement points and the trial(s) where the mean $\mathrm{Oxy}-\mathrm{Hb}$ values significantly decreased compared with the first trial were as follows: $\mathrm{CH} 5$ (the second trial: $p=0.0439$, the third trial: $p$ $=0.0439$ ). There was no significant decrease among three trials in the medial FP and the left lateral FP. In the post-intervention fNIRS measurement, the mean Oxy-Hb values showed the same decreasing tendency as the pre-intervention. The measurement points and the trial(s) where the mean Oxy-Hb values significantly decreased compared with the first trial were as follows: $\mathrm{CH} 4$ (the second trial: $p=0.0439$ ) and $\mathrm{CH} 5$ (the second trial: $p=0.0439$ ) in the lateral FP in the right hemisphere; $\mathrm{CH} 7$ (the second trial: $p=0.0439$ ) in the medial FP in the right hemisphere and $\mathrm{CH} 10$ (the second trial: $p=0.0286$ ) in the medial FP in the left hemisphere; $\mathrm{CH} 12$ (the second trial: $p=0.0286$ ) and $\mathrm{CH} 13$ (the second trial: $p=0.0022$, the third trial: $p=0.0111$ ) in the lateral FP in the left hemisphere (Figure 7).

\subsubsection{Intergroup Comparison of the Maximum $\mathrm{Oxy}-\mathrm{Hb}$ Values between Pre- and Post-Intervention fNIRS Measurements}

Comparison of the maximum Oxy-Hb values between the pre- and post-intervention 
Post-intervention

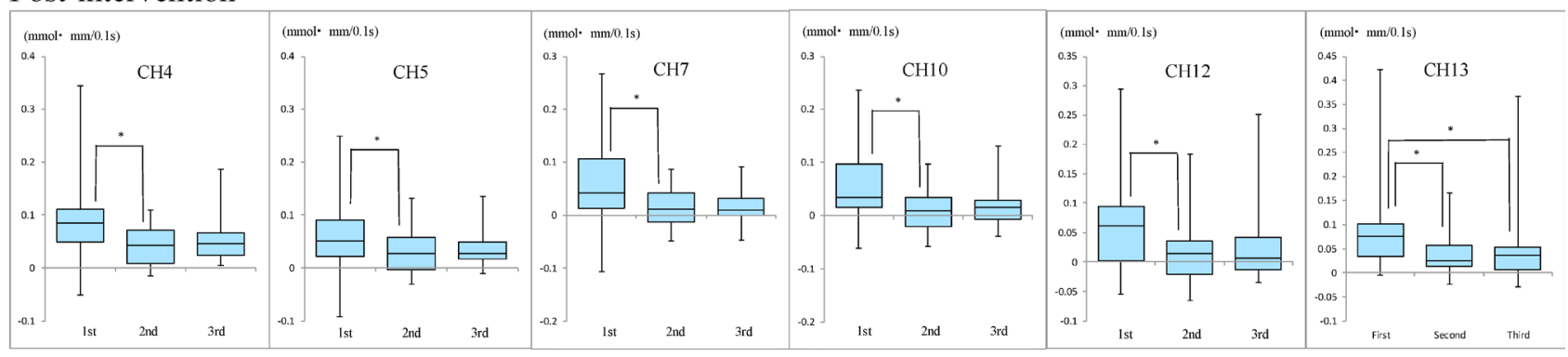

fNIRS measurements by trials by the Wilcoxon Signed-Rank test showed no significant differences as for the first and the second trials. However, as for the third trials, the maximum Oxy-Hb value in the post-intervention measurement was significantly higher at $\mathrm{CH} 4(p=0.0475)$ and $\mathrm{CH} 5(p=0.0386)$ (Figure 8 and Figure 9), while there was no significant difference at other CHs.

\subsubsection{Intergroup Comparison of Mean $\mathrm{Oxy}-\mathrm{Hb}$ Values between Pre- and Post-Intervention fNIRS Measurements}

Comparison of the mean $\mathrm{Oxy}-\mathrm{Hb}$ values between the pre- and post-intervention

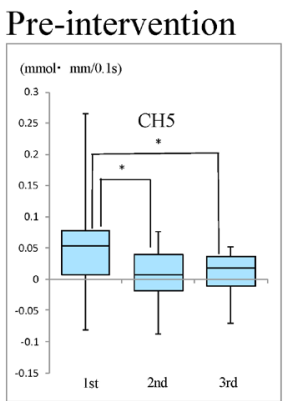

Figure 7. Comparison of the mean $\mathrm{Oxy}-\mathrm{Hb}$ values during the watering task among three trials in the pre- and the post-intervention measurements. A boxplot on the upper column shows the measurement points that demonstrated a significant change in the pre-intervention measurement and boxplots on the lower column show those in the post-intervention measurement: ${ }^{\star} p<0.05$. $\mathrm{CH} 4$ and $\mathrm{CH} 5$ reflect the right lateral FP, $\mathrm{CH} 12$ and $\mathrm{CH} 13$ reflect the left lateral $\mathrm{FP}$. $\mathrm{CH} 7$ reflects the right medial $\mathrm{FP}$ and $\mathrm{CH}$ 10 reflects the left medial FP. The longitudinal axis represents Oxy-Hb concentration.

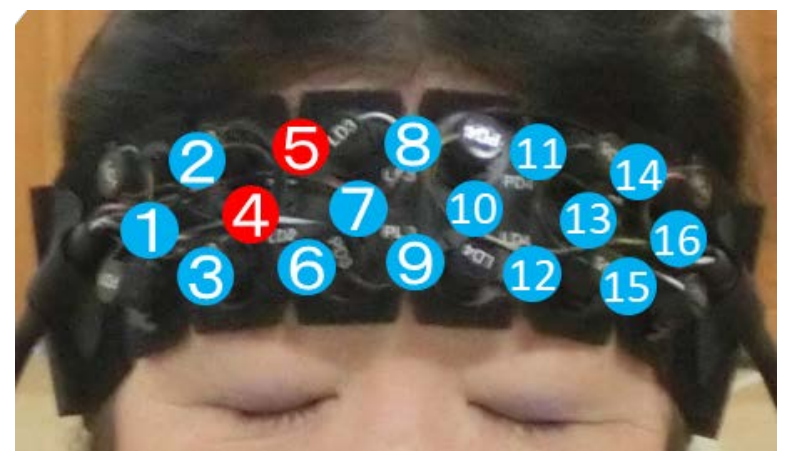

Figure 8. Location of the measurement points where significant higher maximum $\mathrm{Oxy}-\mathrm{Hb}$ values were observed in the post-intervention measurement compared with those in the pre-intervention measurement. Numbered circles represent 16 channels. Two red circled channels four and five show that activation in the right lateral FP was significantly higher than the pre-intervention measurement $(p<0.05)$. There was no significant difference at blue circled channels. 
fNIRS measurements by trials by the Wilcoxon Signed-Rank test was conducted and there was no significant difference between the first trial and the second trial. As for the third trials, the mean Oxy-Hb value in the post-intervention measurement was significantly higher at $\mathrm{CH} 4(p=0.0347)$ and $\mathrm{CH} 5(p=0.0429)$ (Figure 10), while there was no significant difference at other $\mathrm{CHs}$.

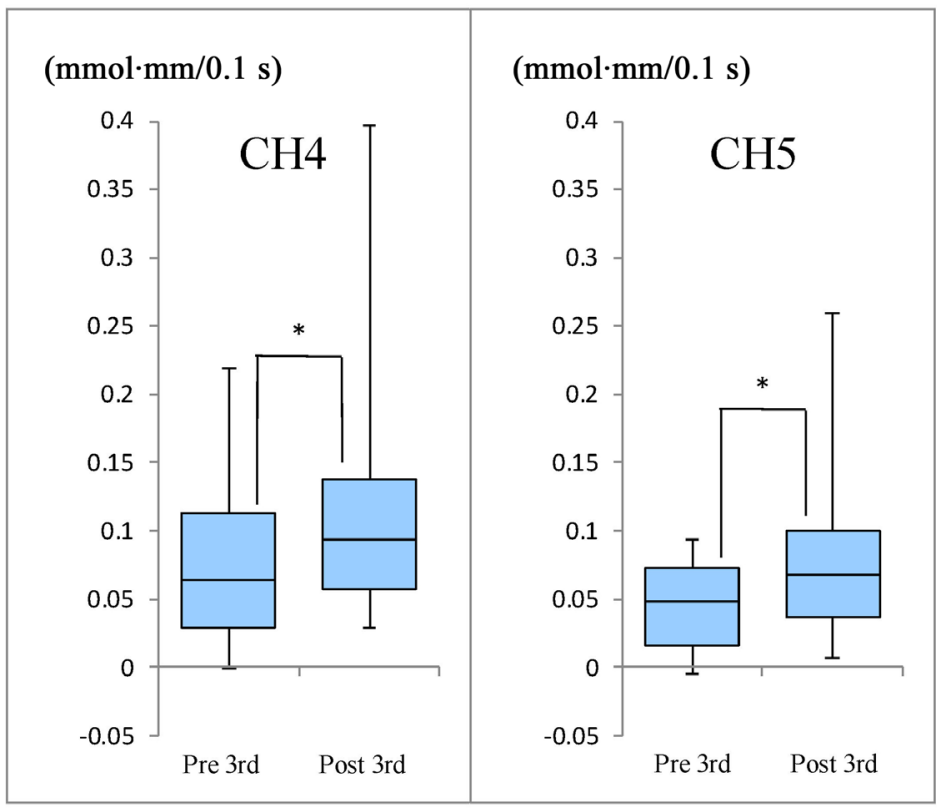

Figure 9. Comparison of the maximum $\mathrm{Oxy}-\mathrm{Hb}$ values during the watering task between the pre- and the post-intervention measurements. $\mathrm{CH} 4$ and $\mathrm{CH} 5$ reflect the right lateral FP. The longitudinal axis represents $\mathrm{Oxy}-\mathrm{Hb}$ concentration: ${ }^{\star} p<0.05$.

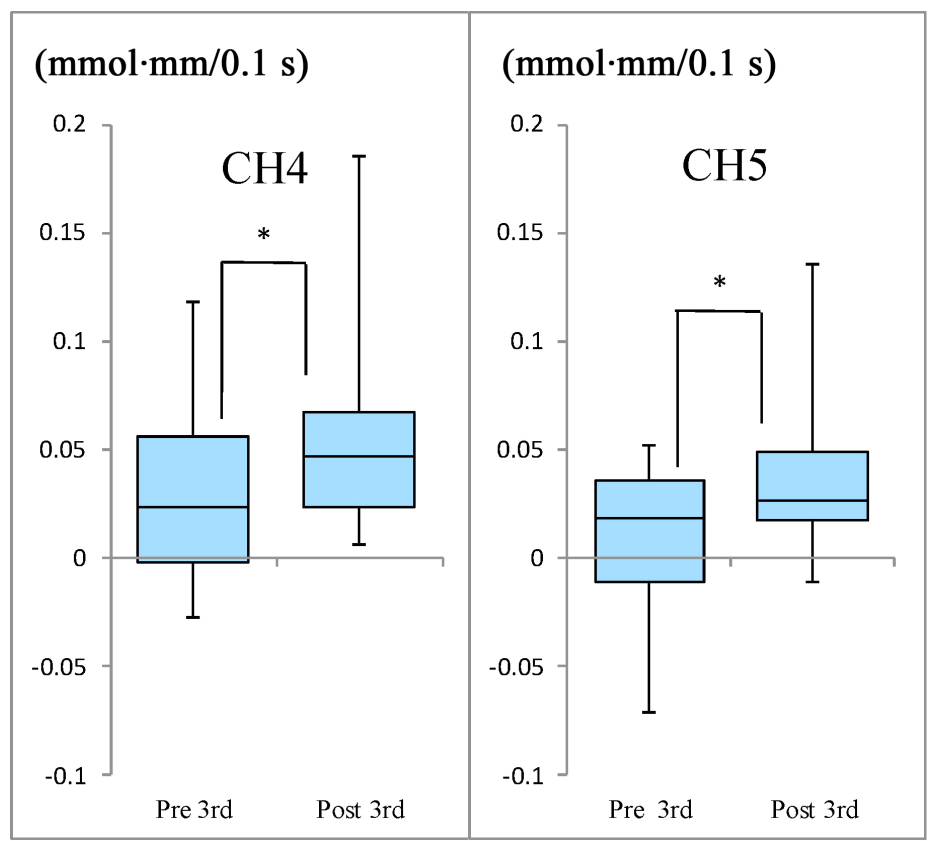

Figure 10. Comparison of the mean $\mathrm{Oxy}-\mathrm{Hb}$ values between the pre-intervention and the post-intervention measurements. The longitudinal axis represents $\mathrm{Oxy}-\mathrm{Hb}$ concentration: ${ }^{*} p<0.05$. CH 4 and $\mathrm{CH} 5$ reflect the right lateral FP. 


\section{Discussion}

\subsection{Improvement of Task Performance}

The participants' task performance showed the similar tendency both in the preand the post-intervention measurements. The task performance in the second and the third trials improved compared with the first trial (Figure 4). In addition, the task performance in the first trial in the post-intervention measurement was higher than that in the pre-intervention measurement (Figure 5). From the above, it can be said that the watering task performance of the older adults improved after three-month intervention.

\subsection{FP Function Required for the Watering Task}

Prior to executing the watering task, the participants were requested to memorize the prescribed way of watering according to the instruction. The watering task consisted of three processes: 1) to water the soil in a cell in a cell tray, 2) to raise the plastic bottle upright and 3) to move to the next cell to water.

During the task performance, attention to visual stimulation such as a cell, a plastic bottle, pouring water, and somatosensory stimulation such as the weight of the plastic bottle containing water and the tilt of the plastic bottle are necessary. Burgess, Simons, Dumontheil, and Gilbert described a function of medial BA10 [Brodmann area 10] as "biasing attention toward current sensory input" [19]. Oxy-Hb values at $\mathrm{CH} 7$ and $\mathrm{CH} 10$ are considered to reflect the medial FP activation due to biasing attention toward current sensory input, namely SO (stimulus-oriented) attention/thought.

Memorizing a set of instructions about how to water, recalling individual action at each process and thinking of the next action to be taken during task performance are presumed to correspond to SI attending [15] or SI thought [16]. The lateral FP is considered to be "activated bilaterally during periods of extended stimulus-independent cognition" [20]. Thus, Oxy-Hb values at $\mathrm{CHs} 4,5$, and 6 in the right hemisphere, and CHs 11,12, and 13 in the left hemisphere seem to reflect the lateral FP activation during SI thought.

Different functions have been reported for the lateral FP in each hemisphere. Gilbert, Frith, and Burgess reported transient activity in the right lateral rostral PFC by a switch between SO and SI thought [16]. In our study, attending to visual and somatosensory stimulation corresponds to SO attention/thought. Switching attention between external stimulation (such as visual and somatosensory stimulation) and internal thought (such as thought of the next action) is considered as switching attention between $\mathrm{SO}$ attention and SI thought. Oxy-Hb values at $\mathrm{CHs} 4,5$, and 6 also reflect the right lateral FP activation during switching attention between $\mathrm{SO}$ attention and SI thought.

Pollmann indicated selectively increased dimension-change-related activation in the left FP for stimulus-driven visual dimension weighting [21]. Changes in the participant's visual weighting during watering are thought to be visual dimension changes: As watering proceeds, the participant's focus moves, within a 
certain sight, to a frame of the cell, the nozzle of the plastic bottle, the running water from the nozzle, and the rising level of water in the cell sequentially. Oxy-Hb values at $\mathrm{CHs} \mathrm{11,12,} \mathrm{and} 13$ also reflect the left lateral FP activation during visual dimension change.

\subsection{Characteristics of the FP Activation during the Watering Task and Task Demands}

Focusing on $\mathrm{CH} 7$ and $\mathrm{CH} 10$ located in the medial FP, the tendency of activation was similar both in the pre- and the post-intervention fNIRS measurements in that the maximum and the mean $\mathrm{Oxy}-\mathrm{Hb}$ values in the first trial were the highest of the three trials. However, as we mentioned in Section 3.2.1 and Section 3.2.2, comparison of the maximum and the mean $\mathrm{Oxy}-\mathrm{Hb}$ values among three trials in the medial FP did not show a statistical decrease in the second and the third trials compared with the first trial in the pre-intervention measurement. This result was the same as that in our previous study where the healthy older adults repeated the watering task five times [11]. On the contrary, in the post-intervention fNIRS measurements, the maximum and the mean Oxy-Hb values showed a statistical decrease in the second trial at both channels compared with the first trial.

The reason why there was no significant change among three trials in the pre-intervention is presumed that the watering task required coincident attention to two types of external input (i.e. visual information, and somatosensory information) and the medial FP activation was necessary even after the second trial to learn a suitable motion for performing the task. However, in the post-intervention measurement after three months (i.e. after putting watering into practice over one thousand times), the participants were well accustomed to the external input during watering. As a result, the medial FP did not need the similar activation any longer to do the same action.

As we mentioned in Sections 3.2.1 and 3.2.2, the activation of the lateral FP showed a similar declining tendency to that of the medial FP: The maximum and the mean $\mathrm{Oxy}-\mathrm{Hb}$ values were the highest in the first trial and decreased in the second and third trials both in the pre- and the post-intervention measurements. The reason why the number of channels showing a significant decrease in activation increased in the second trial in the post-intervention measurement is presumed that the participants got accustomed enough to watering. As a result, they needed less SI cognition for performing the task compared with the pre-intervention measurement.

In spite of a gradual performance improvement during three trials in the preand post-measurements, the FP activation in the second or the third trial showed a declining tendency in both measurements. This indicates that the task demands of cognitive function in watering were not so high. Our results support the idea proposed by Strange, Henson, Friston, and Dolan: "we suggest that the FPPC (fronto-polar prefrontal cortices) is engaged during intentional or explicit 
rule induction but once a rule is learnt, more posterior prefrontal areas mediate rule application" [22].

In addition, we would like to discuss the FP activation related to characteristics of watering: Even if the participants are well accustomed to the watering task after a three-month actual practice (estimated over 1000 times) at home, activation in both the medial and the lateral FP were observed at first during executing the task in the prescribed way another day. This result indicates a possibility that watering in a prescribed, same way may activate the FP whenever the participants start watering.

\subsection{Activation in the Right Lateral FP from the Viewpoint of Age-Related Compensation}

Concerning the reasons of the suppression of activation decline at $\mathrm{CH} 4$ and $\mathrm{CH}$ 5 in the third trials within the post-measurement, first, we discuss a possibility of age-related compensation.

Cabeza and Dennis proposed a model of age-related compensation in PFC activation [23]. This model suggested that mismatch between available cognitive processing resources and task demands triggers the recruitment of reserve neural resources to increase the neural supply. They regarded an increase in neural supply that may enhance cognitive processing as "successful compensation" and the increase in neural supply that may not enhance cognitive processing as "unsuccessful compensation". Is there a possibility that an age-related decline in the lateral FP function over the course of a three-month study period induced a greater activation in the right lateral FP than the pre-intervention measurement to perform the same watering task?

In our study, both in the pre- and the post-measurements, the task performance in the second and the third trials improved compared with the first trial, while the $\mathrm{Oxy}-\mathrm{Hb}$ values during the watering task were the highest in the first trial and decreased in the second and the third trials. This result indicates that the task demand of watering was not high for the participants and the suppression of activation decline in the right lateral FP in the post-measurement was not caused by the mismatch between available cognitive processing resources and task demands. Therefore, it is hard to consider the suppression of activation decline in the right lateral FP as an age-related compensation.

\subsection{A Training Effect of Repeated Watering}

Next, let us discuss a possibility of enhancement of cognitive function by repeating the watering activity.

In this study, the FP activation during the second and the third trials showed a declining tendency. The main reason seems to have been the participants' getting used to the watering process. As a result, the participants needed less FP activation than the first trial. Moreover, in the post-measurement, the task performance improved. 
The increase in the number of times of watering per trial means an increase in the number of switching between SO attention and SI thought within 15 seconds. The result simultaneously means that the ability to continue switching attention between SO attention and SI thoughts throughout a certain period of time required for the task improved. In other words, a three-month watering practice with a plastic bottle at home worked as a training for continual switching attention between external stimuli and internal thought. As a result of the actual watering activity, a favorable change attributable to brain plasticity that the activation in the right lateral FP was hard to decrease was showed confirmed in healthy older adults.

Reijnders, van Heugten, Boxtel indicated in their systematic review on cognitive intervention studies targeting healthy older adults that cognitive training (CT) can be effective in improving various aspects such as executive functioning and attention [24]. Mozolic et al. reported improvement of attention function in their randomized controlled trial (RCT) concerning the effects of CT targeting healthy older adults [25]. CT is defined as "an intervention providing structured practice on tasks relevant to aspects of cognitive functioning" [26]. Gates and Valenzuela also developed an operational definition of CT including components such as repeated practice and use of standardized tasks. They mentioned a trend that "traditional pen-and-pencil exercises are being increasingly replaced by computer-based programs" [27]. Meanwhile, Farzana, Ahuja, and Sreekanth included gardening as one of intellectually stimulating activities effective in cognitive stimulation therapy targeting people with mild to moderate dementia [28].

Watering, one of gardening activities, is not CT intervention using traditional types of CT exercises or computer-based programs. However, our findings suggested that watering has a possibility to contribute to improving attention function of healthy older adults.

\subsection{Changes in the Activated Area in the FP before and after the Intervention}

As we mentioned in Sections 3.2.1 and 3.2.2, some channels that showed decreased activation were different between the pre- and the post-intervention measurements. Jenkins, Brooks, Nixon, Frackowiak, and Passingham pointed out that the neural networks before and after proficiency in skills are different. They mentioned that "Comparing new learning with performance of the prelearned sequence, differences in activation were identified in other areas" [29]. In the course of the three-month continual watering, there might have been some kind of changes in the activated areas.

\subsection{Prediction of the FP Activation during Actual Gardening}

In this study, two possibilities were suggested: even the activity with a small number of steps such as watering, if the activity is continued for a certain period of time, can suppress the decrease in activation in the right lateral FP of older 
adults, and improve task performance (i.e. the number of times per unit time) with frequent switching between SI and SO attention.

Gardening involves many kinds of work such as seeding, weeding, deadheading, harvesting, and so on other than watering. All of them require switching between SO and SI attention. Then, what kind of brain activities in the FP take place during actual gardening? First, let us think about a case where the task-related routine is easily learned. Assuming that pots with well-grown seedlings are evenly arranged, when a person waters them in the same manner every day, the FP activation will occur every time at the beginning of watering. However, the FP activation is deemed to decrease in a relatively short time because the person will get accustomed to the work very soon. Next, let us consider harvesting cherry tomatoes. While a person is harvesting ones, he/she needs to adjust the posture of hands, feet and the whole body according to the situation of the plant in front of him/her. In order to harvest red ripe fruits at the appropriate harvest time, the operation performed for each plant varies every plant in order not to damage other tomatoes within the bunch and/or leaves around the target bunch. In this case, it will take more time to get used to the work than the former case. In addition, a slightly different motion is required for each plant according to the degree of growth of the plant and ripeness of the fruit. As a result, each time the person moves to the next plant to harvest, the FP activation will be repeated as is the case with working at a new task.

\subsection{Prospect}

Some epidemiological studies reported that people who were engaged in gardening had a lower prevalence of Alzheimer's disease [5] [6] [7]. These findings may indicate that continuous physical exercise by gardening contributed to the prevention of Alzheimer's dementia, or that the repetitive motions accompanied by switching between SO attention and SI thought induced the continuous activation in the medial and lateral FP and contributed to the suppression of cognitive decline. Our findings showed a possibility that continuation of watering with only three processes for three months may contribute to the suppression of cognitive decline of healthy older adults. Moreover, continuation of gardening activities combined with different types may provide more effective stimulation to suppress cognitive decline.

In addition, in this study targeting older adults, a continuous gardening activity conduced to improvement in task performance accompanied by suppression of a decrease in the right FP activation. Low activation in the prefrontal area is observed not only in healthy older adults but patients with schizophrenia [30]. In the fields of occupational therapy, horticultural therapy, and green care, there are many cases where agricultural work and gardening programs are provided targeting patients with schizophrenia or people with intellectual disabilities. Further studies to investigate the relationship between the FP activation and task performance when agricultural work or gardening programs including repetitive 
motions are provided to such persons, will be meaningful.

\subsection{Limitation}

In this study, we targeted older adults only. Evidence from younger adults and comparative discussion about the characteristics of the FP activation might be helpful to further figure out the training effect on FP activation by watering.

The fNIRS devise that we used in the present study measures the activation in the prefrontal area such as the whole FP, and portions of the DLPFC, the inferior frontal gyrus (including the orbitofrontal cortex), the pars triangularis Broca's area, and the frontal eye field. As a result, we could not investigate the whole activation of prefrontal area except the FP. Verification using multi-channel fNIRS will also be required in the future research.

\section{Acknowledgements}

This study was supported by Grant-in-Aid for Scientific Research C 15K07298 to Masahiro Toyoda by Japan Society for the Promotion of Science. We are grateful to the Social Welfare Council in Tatsuno City, Hyogo Prefecture, Mr. Hiroshi Takao, the chairperson of Asatomi senior citizen's club and 18 members of the club who participated in this study.

\section{Conflicts of Interest}

The authors declare no conflicts of interest regarding the publication of this paper.

\section{References}

[1] World Health Organization (2019) Dementia Fact Sheets. https://www.who.int/news-room/fact-sheets/detail/dementia

[2] Cherry, K.E., Park, D.C., Frieske, D.A. and Smith, A.D. (1996) Verbal and Picotorial Elaborations Enhance Memory in Young and Older Adults. Aging, Neuropsychology, and Cognition, 3, 15-29. https://doi.org/10.1080/13825589608256609

[3] Kwee, I.L. and Nakada, T. (2003) Dorsolateral Prefrontal Lobe Activation Declines Significantly with Age-Functional NIRS Study. Journal of Neurology, 250, 525-529. https://doi.org/10.1007/s00415-003-1028-x

[4] Belleville, S., Clément, F., Mellah, S., Gilbert, B., Fontaine, F. and Gauthier, S. (2011) Training-Related Brain Plasticity in Participants at Risk of Developing Alzheimer's Disease. Brain, 134, 1623-1634. https://doi.org/10.1093/brain/awr037

[5] Fabrigoule, C., Letenneur, L., Dartigues, J.F., Zarrouk M., Commenges, D. and Barberger-Gateau, P. (1995) Social and Leisure Activities and Risk of Dementia: A Prospective Longitudinal Study. Journal of the American Geriatrics Society, 43, 485-490. https://doi.org/10.1111/j.1532-5415.1995.tb06093.x

[6] Simons, L.A., Simons, J., McCallum, J. and Friedlander, Y. (2006) Lifestyle Factors and Risk of Dementia: Dubbo Study of the Elderly. Medical Journal of Australia, 184, 68-70. https://doi.org/10.5694/j.1326-5377.2006.tb00120.x

[7] Jedrziewski, M.K., Ewbank, D.C., Wang, H. and Trojanowski, J.Q. (2010) Exercise and Cognition: Results from the National Long Term Care Survey. Alzheimer's 
Dementia, 6, 448-455. https://doi.org/10.1016/j.jalz.2010.02.004

[8] Soga, M., Gaston, K.J. and Yamaura, Y. (2017) Gardening Is Beneficial for Health: A Meta-Analysis. Preventive Medicine Reports, 5, 92-99.

https://doi.org/10.1016/j.pmedr.2016.11.007

[9] Toyoda, M., Yokota, Y. and Rodiek, S. (2016) A Motor Programming Task Activates the Prefrontal Cortex More than a Sensitivity-to-Interference Task or an Inhibitory Control Task in Older Adults. Journal of Behavioral and Brain Science, 6, 433-447. https://doi.org/10.4236/jbbs.2016.611040

[10] Toyoda, M. and Yokota, Y. (2016) The Effects of Gardening Activities on Prefrontal Area Measured with Near-Infrared Spectroscopy (NIRS). Acta Horticulturae, 1121, 33-38. https://doi.org/10.17660/ActaHortic.2016.1121.6

[11] Toyoda, M., Yokota, Y. and Rodiek, S. (2017) Gardening May Repeatedly Activate the Frontal Pole. Journal of Behavioral and Brain Science, 7, 464-483. https://doi.org/10.4236/jbbs.2017.710033

[12] Pivik, R.T., Broughton, R.J., Coppola, R., Davidson, R.J., Fox, N. and Nuwer, M.R. (1993) Guidelines for Recording and Quantitative Analysis of Electroencephalo-Graphic Activity in Research Contexts. Psychophysiology, 30, 547-558. http://onlinelibrary.wiley.com/doi/10.1111/j.1469-8986.1993.tb02081.x/full https://doi.org/10.1111/j.1469-8986.1993.tb02081.x

[13] Hoshi, Y., Kobayashi, N. and Tamura, M. (2001) Interpretation of Near-Infrared Spectroscopy Signals: A Study with a Newly Developed Perfused Rat Brain Model. Journal of Applied Physiology, 90, 1657-1662.

http://jap.physiology.org/content/90/5/1657.short https://doi.org/10.1152/jappl.2001.90.5.1657

[14] Sakatani, K., Xie, Y., Lichty, W., Li, S. and Zuo, H. (1998) Language-Activated Cerebral Blood Oxygenation and Hemodynamic Changes of the Left Prefrontal Cortex in Poststroke Aphasic Patients a Near-Infrared Spectroscopy Study. Stroke, 29, 1299-1304. http://stroke.ahajournals.org/content/29/7/1299.short https://doi.org/10.1161/01.STR.29.7.1299

[15] Burgess, P.W., Gilbert, S.J. and Dumontheil, I. (2007) Function and Localization within Rostral Prefrontal Cortex (Area 10). Philosophical Transactions of the Royal Society B, 362, 887-899. https://doi.org/10.1098/rstb.2007.2095

[16] Gilbert, S.J., Frith, C.D. and Burgess, P.W. (2005) Involvement of Rostral Prefrontal Cortex in Selection between Stimulus-Oriented and Stimulus-Independent Thought. European Journal of Neuroscience, 21, 1423-1431. https://doi.org/10.1111/j.1460-9568.2005.03981.x

[17] Spectratech Inc. Press Release (2017) "Hemodynamics Modality Separation (HMS) Method” Developed by AIST Was Adopted for all OEG fNIRS for Research.

https://www.spectratech.co.jp/En/download/Press\%20release\%2020171110.pdf\#sear $\mathrm{ch}=\% 27$ Spectratech+Press+Release $\% 27$

[18] Yamada T., Umeyama, S. and Matsuda, K. (2012) Separation of fNIRS Signals into Functional and Systemic Components Based on Differences in Hemodynamic Modalities. PLoS ONE, 7, e50271. https://doi.org/10.1371/journal.pone.0050271

[19] Burgess, P.W., Simons, J.S., Dumontheil, I. and Gilbert, S.J. (2007) The Gateway Hypothesis of Rostral Prefrontal Cortex (Area 10) Function. In: Duncan J., Phil lips, L. and McLeod, P., Eds. Measuring the Mind: Speed, Control, and Age, Oxford University Press, Oxford, 217-248. https://doi.org/10.1016/j.tics.2007.05.004

[20] Burgess, P.W., Gilbert, S.J. and Dumontheil, I. (2007) Function and Localization within Rostral Prefrontal Cortex (Area 10). Philosophical Transactions of the Royal 
Society B: Biological Sciences, 362, 887-899.

https://royalsocietypublishing.org/doi/full/10.1098/rstb.2007.2095 https://doi.org/10.1098/rstb.2007.2095

[21] Pollmann, S. (2004) Anterior Prefrontal Cortex Contributions to Attention Control. Experimental Psychology, 51, 270-278. https://doi.org/10.1027/1618-3169.51.4.270

[22] Strange, B.A., Henson, R.N.A., Friston, K.J. and Dolan, R.J. (2001) Anterior Prefrontal Cortex Mediates Rule Learning in Humans. Cerebral Cortex, 11, 1040-1046. https://doi.org/10.1093/cercor/11.11.1040

[23] Cabeza, R. and Dennis, N.A. (2012) Frontal Lobes and Aging. Principles of Frontal Lobe Function. 2nd Edition, Oxford University Press, New York, 628-652. https://doi.org/10.1093/med/9780199837755.003.0044

[24] Reijnders, J., van Heugten, C. and Boxtel, M. (2013) Cognitive Interventions in Healthy Older Adults and People with Mild Cognitive Impairment: A Systematic Review. Ageing Research Reviews, 12, 263-275. https://doi.org/10.1016/j.arr.2012.07.003

[25] Mozolic, J.L., Long, A.B., Morgan, A.R., Rawley-Payne, M. and Laurienti, P.J. (2011) A Cognitive Training Intervention Improves Modality-Specific Attention in a Randomized Controlled Trial of Healthy Older Adults. Neurobiology of Aging, 32, 655-668. https://doi.org/10.1016/j.neurobiolaging.2009.04.013

[26] Martin M., Clare L., Altgassen A.M., Cameron M.H. and Zehnder F. (2011) Cognition-Based Interventions for Healthy Older People and People with Mild Cognitive Impairment (Review). Cochrane Database of Systematic Reviews, 1, CD006220. https://doi.org/10.1002/14651858.CD006220.pub2

[27] Gates, N. and Valenzuela, M. (2010) Cognitive Exercise and Its Role in Cognitive Function in Older Adults. Current Psychiatry Reports, 12, 20-27. https://doi.org/10.1007/s11920-009-0085-y

[28] Farzana, F., Ahuja, Y.R. and Sreekanth, V. (2013) Non-Pharmacological Interventions for Enhancing Brain Plasticity and Promoting Brain Recovery: A Review. Research in Neuroscience, 2, 39-49. http://article.sapub.org/10.5923.j.neuroscience.20130203.02.html

[29] Jenkins, I.H., Brooks, D.J., Nixon, P.D., Frackowiak, R.S. and Passingham, R.E. (1994) Motor Sequence Learning: A Study with Positron Emission Tomography. Journal of Neuroscience, 14, 3775-3790. https://doi.org/10.1523/JNEUROSCI.14-06-03775.1994

[30] Takizawa, R., Kasai, K., Kawakubo, Y., Marumo, K., Kawasaki, S., Yamasue, H. and Fukuda, M. (2008) Reduced Frontopolar Activation during Verbal Fluency Task in Schizophrenia: A Multi-Channel Near-Infrared Spectroscopy Study. Schizophrenia Research, 99, 250-262. https://doi.org/10.1016/j.schres.2007.10.025 